\title{
Review
}

\section{Randomized Controlled Trials of Pediatric Massage: A Review}

\author{
Shay Beider ${ }^{1}$ and Christopher A. Moyer ${ }^{2}$ \\ ${ }^{1}$ Integrative Touch for Kids, Beverly Hills, CA and ${ }^{2}$ University of Illinois at Urbana-Champaign, Urbana, \\ IL 61801-4819, USA
}

\begin{abstract}
The existing reviews of massage therapy (MT) research are either limited to infants, adults, or were conducted prior to the publication of the most recent studies using pediatric samples. Randomized controlled trials (RCTs) of pediatric MT are reviewed. A literature search yielded 24 RCTs of pediatric MT, defined as the manual manipulation of soft tissue intended to promote health and well-being in recipients between 2 and 19 years of age. Because RCTs of pediatric MT varied considerably in the amount and types of data reported, quantitative and narrative review methods were both used. Singledose and multiple-dose effects were examined separately. Among single-dose effects, significant reductions of state anxiety were observed at the first session $(g=0.59, P<0.05)$ and the last session $(g=1.10, P<0.01)$ of a course of treatment. Effects for salivary cortisol $(g=0.28)$, negative mood $(g=0.52)$ and behavior $(g=0.37)$ were non-significant. Three of eleven multiple-dose effects were statistically significant. These were trait anxiety $(g=0.94, P<0.05)$, muscle tone $(g=0.90$, $P<0.01)$ and arthritis pain $(g=1.33, P<0.01)$. Results of studies not permitting effect size calculation were judged to be generally consistent with quantitative results. MT benefits pediatric recipients, though not as universally as sometimes reported. Numerous weaknesses endemic to MT research (e.g. low statistical power, frequent failure to report basic descriptive statistics) are identified, and recommendations for future pediatric MT research are discussed.
\end{abstract}

Keywords: CAM - child - comfort - health care - integrative medicine - kid - pain management quality of life - touch

\section{Introduction}

\section{Background}

Massage therapy (MT) is one of the most widely used complementary and alternative medicine (CAM) therapies in the United States according to National Health Interview Survey data. It is estimated that consumers spend between 2 and 4 billion dollars on 75 million visits to massage therapists annually (1). Studies indicate that parents are making increasing use of CAM therapies, including MT, for their children. One study found that $33 \%$ of parents reported using CAM for their child within the past year, with MT being

For reprints and all correspondence: Shay Beider, Integrative Touch for Kids, Founder and Executive Director, 8306 Wilshire Boulevard \#530, Beverly Hills, CA 90211-2304, USA. Tel: +1-323-973-0203;

E-mail: shay@integrativetouch.org one of the most popular therapies (2). Another study shows that families of children with special health care needs are almost twice as likely to have used CAM for their child (3). CAM use is now prevalent, even in many traditional medical settings [e.g. $49 \%$ of university-affiliated pain management centers in the US and Canada offer MT (4)], yet pediatricians and other health care professionals are often not informed about the CAM therapies that are being used by their patients (2). This may be especially true for pediatric CAM, where survey results indicate that $81 \%$ of parents currently using CAM for their child wanted to discuss it with their pediatrician, but only $36 \%$ did (5). For CAM to truly be integrated into the health care system, it needs to be openly discussed and recognized for its value, particularly in the area of palliative care (6). A scientific understanding of CAM therapies, such as pediatric MT, will permit a greater understanding of the value of this type of therapy. 
Numerous studies of MT for children and young adults have been conducted. These include randomized controlled trials (RCTs), evaluation studies, descriptive case studies and reviews. However, the existing reviews of MT research are either limited to adult participants, limited to infants, were conducted prior to the publication of the most recent studies using pediatric samples, or neglected to quantify results. Ottenbacher and colleagues (7) quantitative review of tactile stimulation for infants and young children, published nearly 20 years ago, found statistically significant beneficial outcomes for five of the six categories examined (these were motor/reflex, cognitive/language, social/personal, physiological and overall development; visual/auditory was nonsignificant). Field (8) conducted a narrative review, including research on persons of all ages, and concluded that MT had a multitude of condition-specific effects in addition to consistently providing reductions of anxiety, depression and levels of stress hormones (most notably cortisol) that were observed across studies. The two most recent reviews are quantitative reviews that focus specifically on either infants or adults. A Cochrane review systematically examines MT for preterm and low birth-weight infants, and reaches the negative conclusion that 'there is insufficient evidence of effectiveness to warrant wider use of preterm infant massage' (9). The quantitative review of MT performed on adults confirmed (and quantified) some of Field's conclusions while disputing others (10). Substantial reductions resulting from multiple sessions of MT were noted for depression $(g=0.62, P<0.01)$ and trait anxiety ( $g=0.75, P<0.01)$, while MT's effect on cortisol levels was not statistically significant $(g=0.14)$. While this review supported the value of MT by quantifying its ability to substantially reduce symptoms of psychological distress in adults, taken together this set of findings called into question some of the theories most often invoked to explain the benefits of MT, especially the theory that MT benefits recipients primarily by activating the parasympathetic nervous system (10).

\section{Current Review}

While the latest quantitative reviews increase our understanding of MT and indicate new directions for research, they neglect MT performed on pediatric samples. The current review examines MT's effects in pediatric samples (defined here as studies where the mean age of participants was between 2 and 19 years), with a focus on RCTs. While some have argued against using RCTs to understand CAM modalities such as MT (11), RCTs represent the state-of-theart for establishing cause and effect relationships in treatment research because they are the most effective study design for ruling out alternate (i.e. non-treatment) explanations for observed effects, including spontaneous recovery, placebo effects and statistical regression (12).

Though there have been some new pediatric MT studies published since the reviews by Ottenbacher et al. (7) and Field (8), the number of RCTs that examine pediatric MT is still quite small. In addition, a weakness of existing MT research is that, more often than not, studies in this area do not include the minimal statistical detail necessary to calculate effect sizes. For these reasons, a full meta-analytic treatment of MT research with pediatric samples is not possible. However, consistent with Rosenthal's observation that a narrative literature review can only be improved by the addition of a simple, descriptive quantitative analysis (13), we quantified results where possible, and include these results in combination with a narrative review of other RCTs. In some cases, the present findings are being compared with analogous findings in the adult literature. When these comparisons are made, we are referring to the adult meta-analysis conducted by Moyer et al., unless otherwise stated (10).

\section{Operational Definition}

A notable challenge in reviewing MT studies is that there are many forms of MT in practice. The American Massage Therapy Association (AMTA) defines massage as 'manual soft tissue manipulation, [including] holding, causing movement, and/or applying pressure to the body' (14). As written, this very broad definition includes numerous MT approaches commonly used in clinical practice that are relevant to the current review, but could also include rare forms of medical massage (e.g. optic nerve massage (15), light compressive massage for congenital dacryocystocele (16), cardiac massage (17)), that are outside the intended scope of this review. For this reason, we focus on forms of MT that are consistent with traditional Swedish styles of massage. Swedish massage uses five main strokes to stimulate the circulation of blood through the body; petrissage (kneading), effleurage (stroking), friction, tapotement (tapping) and vibration. For the purposes of this review, MT is typified by the manual manipulation of soft tissue, performed by a person other than the recipient, intended to promote health and well-being. This operational definition allows a range of MT styles to be included in this review. Studies vary on many details, including the amount of clothing worn by recipients, whether a massage chair or massage table was used, whether MT took place in a clinical setting or at home, and whether MT was performed by a person with full, partial, or no training as a massage therapist. Studies also vary in which anatomical regions are massaged. Despite all these variations, it is reasonable to expect that there will be some consistent outcomes that result from MT. Eventually, as a scientific understanding of MT grows, studies that examine the importance of these variations will be advisable, but currently the questions of greatest interest are at a more fundamental level.

\section{Types of Effects}

MT effects can be divided into single-dose and multiple-dose. Single-dose effects include MT's influence on psychological or physiological states that are transient in nature and that might reasonably be expected to be influenced by a single session of MT. Multiple-dose effects are restricted to MT's influence on variables that are considered to be more enduring, 
or that would likely be influenced only by a series of MT sessions performed over a period of time, as opposed to a single dose. Frequently, both single- and multiple-dose effects are examined in the same study. One example is a study of MT for autistic children that examined the single-dose effect of MT on salivary cortisol (immediately prior to, and immediately following, an individual session of MT) and the multipledose effect of MT on depression (at the beginning of, and at the conclusion of, a sequence of MT sessions over time) (18). A second example is a study that evaluated children's distress during burn treatment, which included the single-dose effect of MT for state anxiety and the multiple-dose effect of MT for depression (19). Typically, studies include the terms 'shortterm effect' and 'long-term effect' to indicate single- and multiple-dose effects, respectively. Our decision to use the single-dose and multiple-dose terminology is motivated by the desire to prevent any confusion that may arise related to how long an effect may last following the termination of treatment. None of the studies in the current review examine whether any MT effects last beyond the final day on which a participant receives treatment, making the use of the term 'long-term effect' potentially misleading.

The potential benefits of MT can be further classified according to whether they are primarily affective, physiological or behavioral in nature. Affective refers to effects most closely associated with the recipients' feelings and emotions. Physiological effects are those concerned with recipients' vital organismic processes. Behavioral effects are those related to the recipients' observable responses to their environment. Study results reviewed here will first be separated by the single-dose versus multiple-dose distinction, then further categorized into affective, physiological and behavioral dimensions.

\section{Methods}

\section{Literature Search}

A literature search was conducted by the first author (S.B.) using the keywords massage, child and pediatric to search the MEDLINE, LexisNexis, CINAHL and PsycInfo databases. We checked the NIH CRISP Database to search for other publicly funded studies currently in progress. MT researchers were also contacted to obtain studies that were unpublished, in press or otherwise not found by means of database searches. With the introduction of the first Massage Therapy Research Conference in Albuquerque, New Mexico (43), leaders in the field of massage research were readily contacted. We used this opportunity to access current unpublished pediatric massage studies. Studies obtained by these methods were inspected to ensure that they examined a form of MT consistent with the present study's operational definition of MT performed on a pediatric sample. Application of these criteria yielded 24 RCTs of MT with a pediatric sample. These studies, along with important details, are listed in Table 1.

\section{Statistical Analysis}

For studies that provided sufficient data, between-groups comparisons on variables of interest were converted to Hedges' $g$ effect size by the second author (C.A.M.). Hedges' $g$, calculated as (Group mean 1 - Group mean 2)/Pooled SD, estimates the number of standard deviations that the average member of a treatment group differs from the average member of a comparison group for a given outcome. Hedges' $g$ was selected over Cohen's $d$, a similar standardized mean difference effect size. This choice was made for two reasons. First, in some cases the original study data could only be converted to $g$. Second, using $g$ makes the results of the current review as consistent as possible with the existing metaanalysis of MT effects for adults.

In cases where a study employed more than one measure to examine the same outcome variable, results of multiple measures completed by participants or by blinded observers (but not those completed by non-blind observers) were standardized and then averaged, yielding one effect size per variable for each study. Similarly, if a study examined the immediate effects of more than one application of treatment, the results of the multiple applications or assessments were standardized and, when similar in magnitude, averaged in order to calculate a single effect size for that study. In the single case where these assessments clearly differed in a systematic way (i.e. state anxiety, where the effects of a final session were always larger than the effects of the initial session; paired sample $t(3)=4.46$, one-tailed $P<0.02$ ), separate effects were calculated for each timepoint. Effect sizes were coded such that positive values, for any variable, indicate a more desirable outcome (e.g. a reduction in anxiety) for the participants who received MT. Individual study effect sizes were subjected to a correction for small sample bias, then weighted by their inverse variance and averaged to generate a mean effect size for each outcome variable (20). All effect sizes were calculated according to a random effects model of error estimation. Statistical significance of the mean effect sizes was assessed by calculating the $95 \%$ confidence interval for the population parameter. A significance level of 0.05 or better is inferred when zero is not contained within the confidence interval.

\section{Results}

Of the 24 RCTs, only 9-accounting for a total of 200 participants-provided sufficient data for their results to be systematically quantified. Table 2 lists mean effect sizes for 16 outcome variables $(g)$, as well as the number of studies contributing to each effect size $(k)$, the total number of participants contributing to each effect size $(N)$ and 95\% confidence intervals. Five of these sixteen effect sizes were statistically significant. For the single-dose effects category, these included state anxiety at the first session $(g=0.59, P<$ $0.05)$ and at the last session $(g=1.10, P<0.01)$ of a course of treatment. Effects for salivary cortisol $(g=0.28)$, 


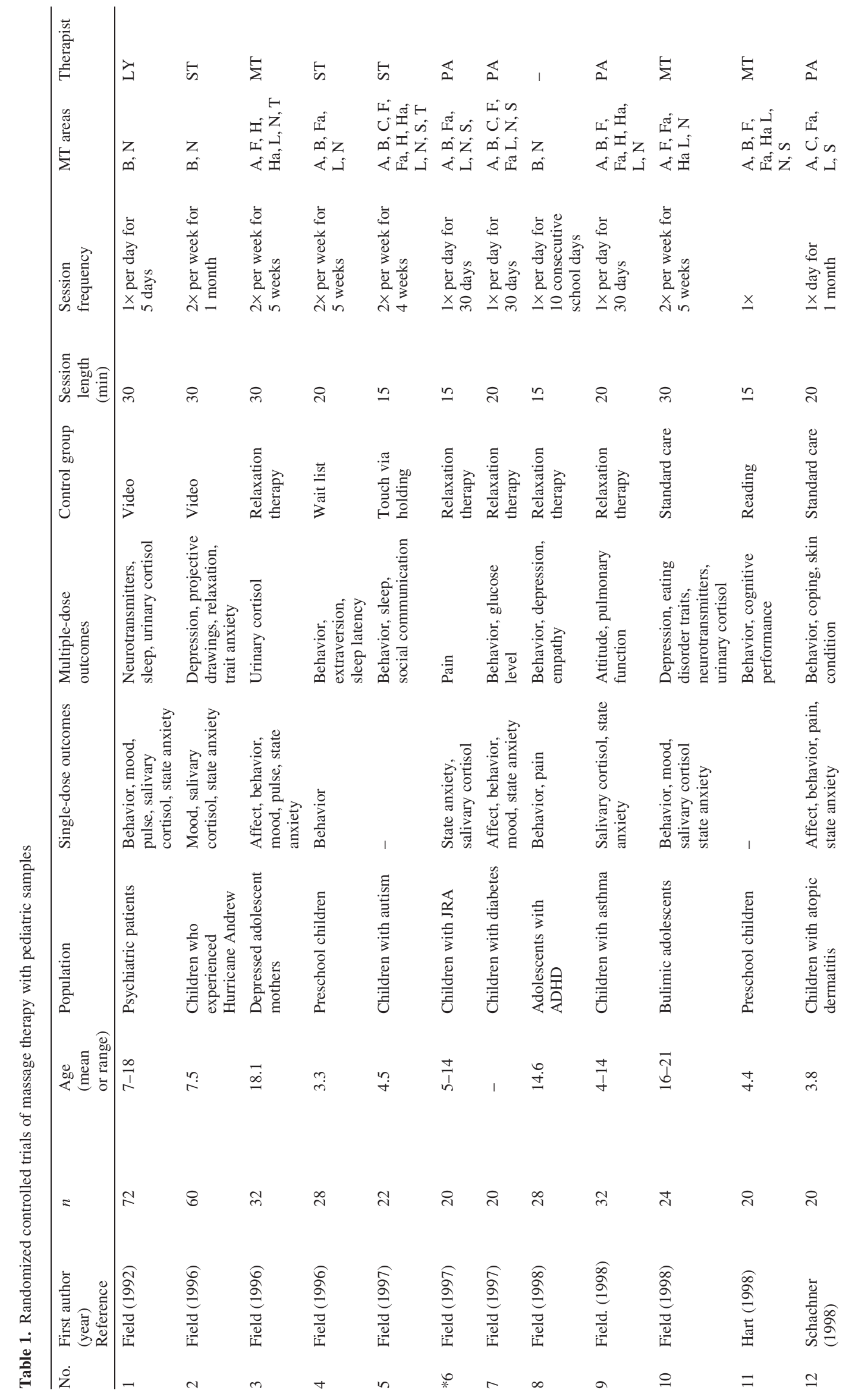




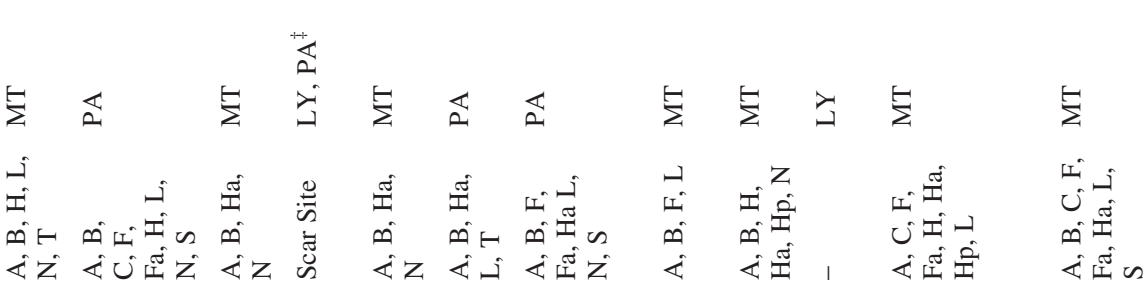

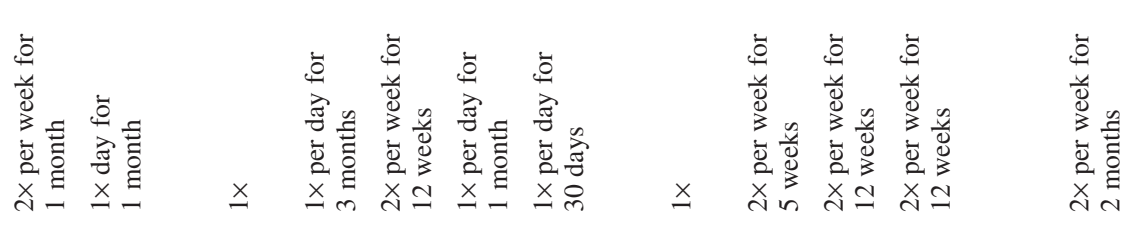

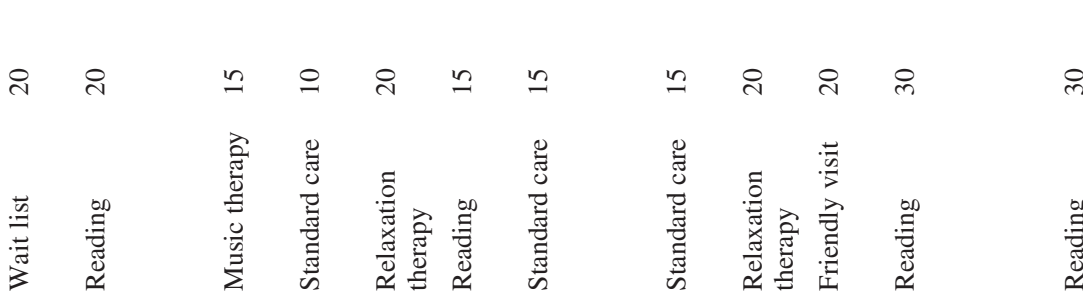

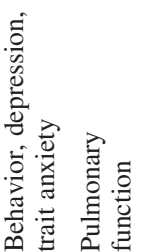

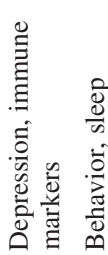

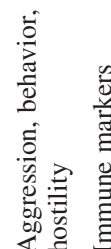

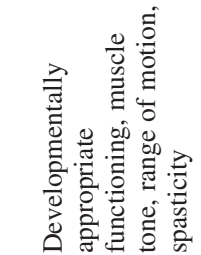

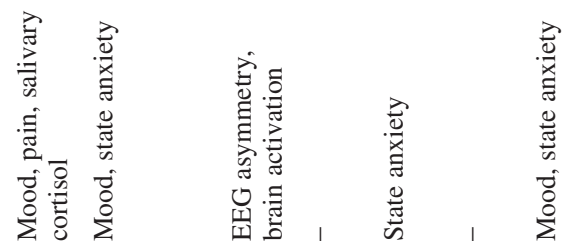

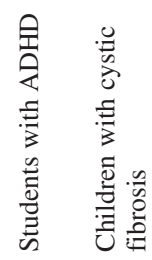

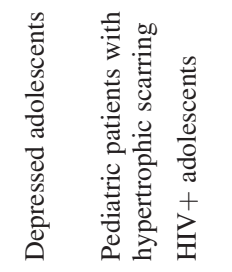

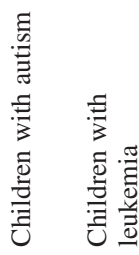

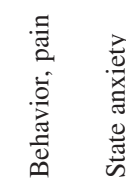

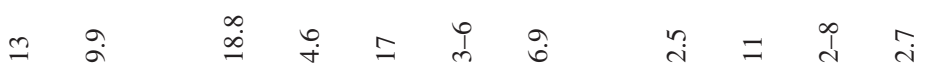

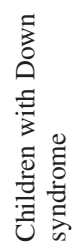

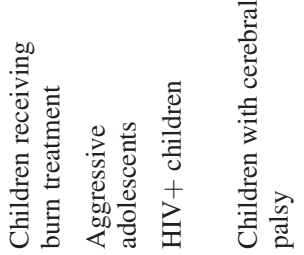

吾

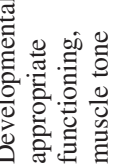

告

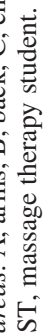

$\Xi$

䓍

a

产客

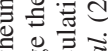

政

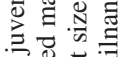

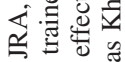

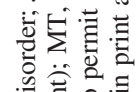

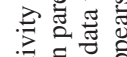

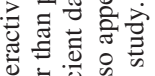

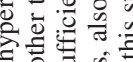

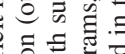

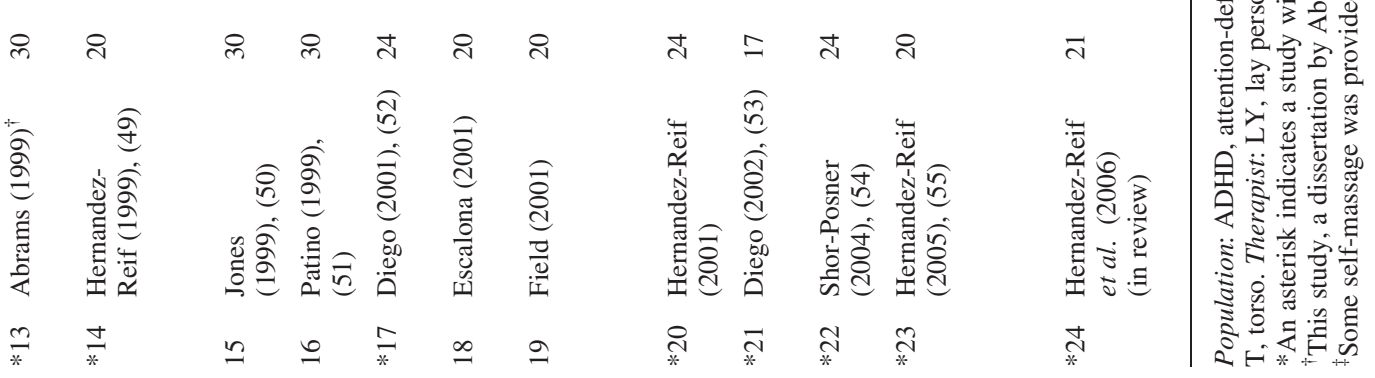


Table 2. Mean effect sizes $(\mathrm{g})$ by outcome variable

\begin{tabular}{lcccr}
\hline Outcome variable & $k$ & $N$ & $g$ & \multicolumn{1}{l}{$95 \%$ CI } \\
\hline Single-dose effects & & & & \\
$\quad$ State anxiety, first session & 4 & 81 & $0.59 *$ & $0.15,1.04$ \\
$\quad$ State anxiety, last session & 4 & 81 & $1.10^{* *}$ & $0.64,1.57$ \\
$\quad$ Negative mood & 2 & 50 & 0.52 & $-0.05,1.10$ \\
Salivary cortisol & 2 & 50 & 0.28 & $-0.27,0.84$ \\
$\quad$ Behavior & 1 & 24 & 0.37 & $-0.43,1.35$ \\
Multiple-dose effects & & & & \\
Depression & 2 & 54 & 0.48 & $-0.06,1.02$ \\
Trait anxiety & 1 & 30 & $0.94 *$ & $0.20,1.68$ \\
Arthritis pain & 1 & 20 & $1.33 * *$ & $0.37,2.29$ \\
Muscle tone & 2 & 41 & $0.90^{* *}$ & $0.23,1.57$ \\
Range of motion & 1 & 20 & 0.31 & $-0.57,1.19$ \\
Immune measures & 2 & 48 & 0.06 & $-0.52,0.63$ \\
Pulmonary function & 1 & 20 & 0.47 & $-0.41,1.35$ \\
Developmental functioning & 2 & 41 & 0.24 & $-0.38,0.86$ \\
Spasticity & 1 & 20 & 0.26 & $-0.62,1.14$ \\
Hostility & 1 & 17 & -0.85 & $-1.85,0.15$ \\
Classroom behavior & 1 & 30 & 0.66 & $-0.07,1.39$ \\
\hline
\end{tabular}

Note: A positive $g$ indicates a reduction for any outcome variable. CI, confidence interval.

$* P<0.05$. $* * P<0.01$.

negative mood $(g=0.52)$ and behavior ( $g=0.37)$ were nonsignificant. Only 3 of the 11 multiple-dose effect sizes were statistically significant. These were trait anxiety $(g=0.94, P<$ $0.05)$, arthritis pain $(g=1.33, P<0.01)$ and muscle tone $(g=$ $0.90, P<0.01)$.

Of the 24 RCTs, 15 (accounting for 458 research participants) do not report sufficient data to permit effect size calculation, a frequent problem in MT research that makes objective interpretation of results difficult. Nevertheless, by judiciously comparing the scant data presented in this subset of studies with the objective data previously summarized, it should be possible to see if there are any dramatic contrasts among the findings. These interpretations, within the context of the more objective findings, appear in the results categories that follow.

\section{Single-Dose Effects}

\section{Affective Dimension}

State anxiety. Field et al. have conducted several pediatric MT studies where anxiety is an outcome measure. These studies date back to 1992 when MT was applied to a group of pediatric psychiatric patients (21). Four studies with reportable effect sizes (No.'s 6, 14, 17 and 21 in Table 1), using a total of 81 participants, compared MT with either relaxation therapy or a reading comparison group with state anxiety-a momentary emotional reaction consisting of apprehension, tension, worry and heightened autonomic nervous system activity (22) — as a dependent variable. Three of the studies (No.'s 14, 17 and 21) used the State Trait Anxiety Inventory (two specify the child version). One study (No. 6) of MT for children with juvenile rheumatoid arthritis used a behavior observation of the child's anxiety level performed by a blinded rater. MT consistently reduced state anxiety in these studies. This single-dose effect was unique in that it is significantly larger at the second timepoint, so we examined these separately. Results of a first session of MT yield a statistically significant effect, $g=0.59$ $(95 \% \mathrm{CI}=0.15,1.04)$. The effect at the last session is even greater, $g=1.10(95 \% \mathrm{CI}=0.64,1.57)$. These results are depicted graphically in Fig. 1. Possibly, the substantially larger effect occurring at the last session of treatment may be the result of participants' increasing comfort with MT (or with the massage therapist) over the course of time, or the effect may be related to MT's potential to reduce trait anxiety over a course of treatment (8). These pediatric results are consistent with the same effect found for adults, where this effect has been estimated as $g=0.37(95 \% \mathrm{CI}=0.14,0.59)$. Possibly, the larger effect for the pediatric samples examined here reflects a greater treatment aptitude for this population; however, with such wide confidence intervals, this is only speculation.

Studies where state anxiety was a dependent variable but effect sizes could not be calculated include samples of children who experienced Hurricane Andrew (23), depressed adolescent mothers (24), and children and adolescents with a range of illnesses including diabetes (25), atopic dermatitis (26), asthma (27), bulimia (28) and leukemia (29). The most commonly used measures across these studies were the State Trait Anxiety Inventory for Children (STAIC) and the State Trait Anxiety Inventory (STAI) for adolescents. A study of children who experienced Hurricane Andrew assessed both the single-dose effects of state anxiety and the multiple-dose effects of trait anxiety. These studies are consistent with the previously reported effect sizes, in that MT appears to decrease state anxiety. The MT in all of these studies was conducted either daily or twice weekly over a treatment period of 30-45 days. MT sessions generally lasted between 20 and $30 \mathrm{~min}$, the one exception being the leukemia study where MT sessions were $15 \mathrm{~min}$ in duration. Four of these studies trained parents to provide MT directly to their child and the remaining three either used trained massage therapists or massage students.

Mood. Mood, which may be defined as 'transient episodes of feeling or affect' (30), has frequently been an outcome measure in pediatric MT studies. Study populations have included depressed adolescent mothers, and children and adolescents with cystic fibrosis, leukemia and bulimia. Two studies (No.'s 13 and 14 in Table 1), using a total of 50 participants, compared MT with either a reading or wait-list control to examine MT's effect on mood. One of these studies (No. 14) used the Profile of Mood States (POMS) (31) depressed mood subscale. The second study (No. 13) used a faces scale and a modification of the Children's Pain/Fear Thermometer Rating Scale. Taken together, these studies yield a non-significant effect, $g=0.52(95 \% \mathrm{CI}=-0.05,1.10)$ that is consistent with the results found for adult recipients ( $g=0.34,95 \%$ CI $=-0.08,0.76)$. These positive but 


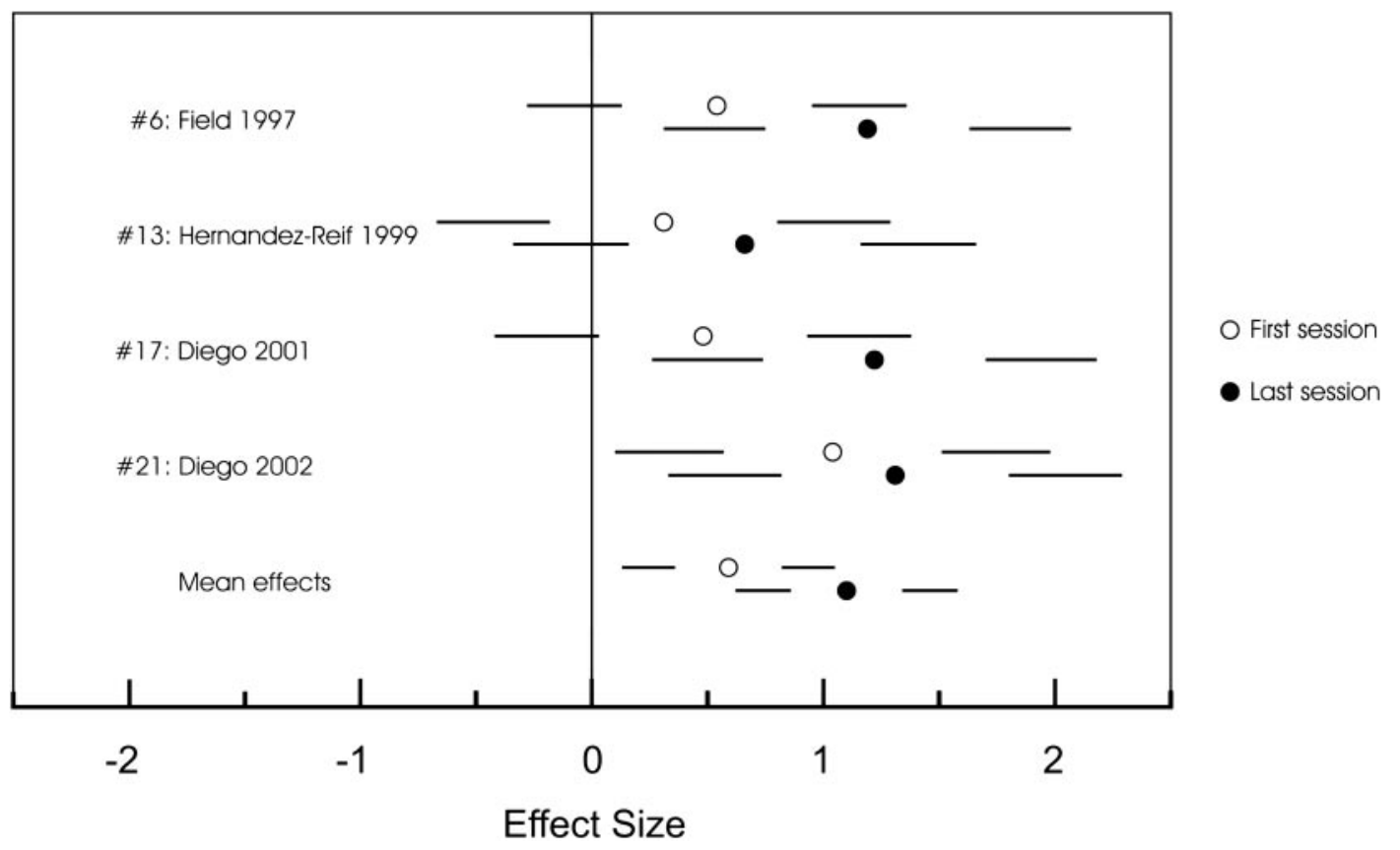

Figure 1. Single-dose effects ( $g$ and 95\% CI) of MT on state anxiety obtained at first sessions and last sessions of treatment.

non-significant results suggest at least four possibilities. One is that the tools that have been used to measure mood in these studies are not precisely capturing overall mood, but are being affected by correlates of mood such as depression, pain or fear. A second possibility is that MT's modest effect on mood has not been examined with sufficient statistical power, resulting in wide confidence intervals. The third is that MT's positive effect on mood is affected by a moderator variable that has not yet been examined (e.g. recipient's comfort with MT; the existence of a therapeutic bond between the recipient and provider, etc.). Finally, the possibility that MT does not specifically have a positive effect on mood cannot be definitively ruled out.

\section{Physiological Dimension}

Salivary cortisol. Two studies (No.'s 6 and 13 in Table 1), using a total of 50 participants, compared MT with either relaxation training or a wait-list control to examine MT's single-dose effect on cortisol-a stress hormone associated with activation of the sympathetic nervous system in response to certain kinds of stressors. In both studies, salivary cortisol [but not urinary cortisol, which less accurately captures shortterm stress responses (32) most likely to respond to single-dose MT] was sampled pre- and post-MT session, with a delay of 20-30 min after the session, because salivary cortisol samples reflect responses to stimulation occurring $\sim 20 \mathrm{~min}$ prior to collection. Both studies also took into account the diurnal cortisol cycle, characterized by an increase in secretory activity following awakening and a declining trend over the course of the day (33). Combined, these studies yield a nonsignificant reduction, $g=0.28$ (95\% CI $=-0.27,0.84)$ of salivary cortisol for the participants receiving MT in comparison to controls. This finding contrasts Field's assertion that reductions in cortisol level are one of MT's most reliable effects, but is consistent with meta-analytic findings based on adult samples, where cortisol effects were small and nonsignificant ( $g=0.14,95 \% \mathrm{CI}=-0.10,0.38)(10)$. Based on the available evidence, MT's single-dose effect on cortisol levels appears to be small, and possibly zero.

\section{Behavioral Dimension}

Distress behaviors. One study (No. 20 in Table 1) compared MT with standard care for children receiving burn treatment, and examined the children's distress behaviors before and during this painful procedure. The Children's Hospital of Eastern Ontario Pain Scale (34) was used to code distress behaviors before and during a dressing change (35). Six behavior categories were assessed including cry, facial, verbal, torso, touch and legs. The MT group showed only an increase in torso movements during the dressing change, whereas the control group showed an increase in five out of the six distress behaviors. Combined observational ratings made by nurses, who were blind to the group to which the children were assigned, favored MT, $g=0.37$ (95\% CI $=-0.43,1.17)$. Though this effect favors MT, the wide confidence interval, which may be partially or wholly attributable to the small sample $(n=24)$, makes this result difficult to interpret.

In several other studies with behavioral outcomes where effect sizes could not be calculated, researchers reported improvements in fidgetiness, activity, vocalization and cooperation. The most commonly used instrument in these studies is a Behavior Observation Scale that was first used to assess 
behavior after relaxation therapy classes (36). Using this measure, behavior is observed three times during the $30 \mathrm{~min}$ prior to MT, during MT itself and during the $30 \mathrm{~min}$ after MT. Because the effect size data are inconclusive and researchers report positive behavioral improvements resulting from MT, further study on distress behaviors may be warranted.

\section{Multiple-Dose Effects}

\section{Affective Dimension}

Depression. Depression, including motivational and cognitive deficits, vegetative signs, and disruptions in interpersonal relationships beyond those expected from ordinary unhappiness or poor mood (37), has been examined in pediatric MT studies, albeit with small samples. Two studies (No.'s 13 and 17 in Table 1), using a total of 54 participants, examined whether MT would reduce depression in comparison to either a wait-list control or to progressive muscle relaxation. The first study (No. 13) used the Children's Depression Inventory-Short Form, and was the only study reviewed to use that measure. It is an abbreviated version of a widely used self-report measure of depression for children and adolescents. The second study (No. 17) used the Center for Epidemiological StudiesDepression scale, which was also used in several studies that did not permit effect size calculation. Though the mean effect favored MT, $g=0.48$, this effect is not statistically significant (95\% CI $=-0.06,1.02)$. This result is inconclusive, but promising given that the confidence interval approaches significance, and also because the estimated effect is not greatly different from statistically significant reductions of depression found in adult samples $(g=0.62,95 \% \mathrm{CI}=0.37$, 0.88 ). It is also generally consistent with the conclusions reached by the authors of studies that did not permit effect size calculation. Still, the possibility that MT does not reduce depression for pediatric recipients to the extent it does in adults cannot be ruled out. At this point, further study on MT for pediatric depression is needed.

Trait anxiety. One study (No. 13 in Table 1), using a sample of children and adolescents with attention-deficit hyperactivity disorder, examines MT's effect on trait anxiety, a dispositional, internalized proneness to be anxious (38), with enough detail to permit effect size calculation. This study, which also appears in the adult MT meta-analysis, uses the Revised Children's Manifest Anxiety Scale to compare MT with a wait-list condition and yields a large, statistically significant effect ( $g=0.94,95 \% \mathrm{CI}=0.20,1.68)$ that is consistent MT's anxiolytic effects demonstrated in numerous studies with adult recipients. This result is consistent with author claims in the 'Hurricane Andrew' study, where improvements in children's trait anxiety at the end of the MT treatment are reported. Reduction of trait anxiety, resulting from a course of MT sessions, is certainly worthy of further study in pediatric populations.

Pain. Four pediatric studies have been conducted that include pain as an outcome measure. Three of these studies used the Happy Faces Scale while only one study, a study of children with juvenile rheumatoid arthritis (and the only study with sufficient data for calculating effect sizes), used the Varni/Thompson Pediatric Pain Questionnaire. This study (No. 6 in Table 1) assessed pain in three ways-child self report, parent report and physician report. The children and a pediatric rheumatologist, who was blind to group assignment, generated reports of pain reduction that were consistent; parents' ratings were omitted from effect size calculation due to their non-blind status and proneness to bias. In comparison to a relaxation therapy group, MT yielded a large, statistically significant pain reduction $(g=1.33,95 \% \mathrm{CI}=0.37,2.29)$. This very large effect, in contrast with other studies that have shown mixed results for pain reduction resulting from MT, suggests that MT may be particularly well-suited to pain reduction for children with this condition.

Three RCT's that do not permit effect size calculation looked at MT's impact on pain, and concluded that children experienced reductions of pain resulting from MT. Samples in these studies included children who experienced Hurricane Andrew (23), adolescents with attention-deficit hyperactivity disorder (39) and children with atopic dermatitis (26). These reports, combined with the findings in the juvenile rheumatoid arthritis study, indicate that MT for pain reduction in pediatric recipients is worthy of further examination.

\section{Physiological Dimension}

Muscle tone. Two recent studies that assess muscle tone (No.'s 23 and 24 in Table 1) yield $g=0.90$ (95\% CI $=0.23$, 1.57). The first examines MT for children with cerebral palsy who received $30 \mathrm{~min}$ of MT 2 times per week for 12 weeks. This study also assesses spasticity, motor functioning, facial expressions and limb activity. The second study, of children with Down syndrome provided 30 min of MT 2 times per week for 8 weeks. Children's development, and fine and gross motor functioning, was evaluated. Both studies used the Arms, Legs and Trunk Muscle Tone Scale (ALT Muscle Tone Scale), which was designed during the pilot phase of the Down syndrome study. There is modest support that MT improves muscle tone, though it must be pointed out that the two studies diverge greatly in their individual results. Study number 23 in Table 1 had virtually no effect, while study number 24 had a huge effect. It must be noted that because these results come from a newly developed measure, the validity of the measure is not yet well-established. This may account for the divergent results across studies.

Range of motion. One study (No. 23 in Table 1), with a sample of children suffering from cerebral palsy, yields nonsignificant improvements in range of motion, $g=0.31(95 \%$ $\mathrm{CI}=-0.57,1.19)$. The study notes that right and left hip extension, but not abduction, improved. Study authors note that increased muscle tone may have led to an increase in range of motion, but this result, based on a single study with 20 participants, is inconclusive. 
Immune measures. Two studies (No.'s 17 and 22 in Table 1) examined markers of immune system functioning in $\mathrm{HIV}+$ adolescents or children. Though study authors are quick to point out that there were some within-group effects for those who received MT, a between-groups analysis that compares MT recipients with controls yields no effect ( $g=0.06,95 \%$ $\mathrm{CI}=-0.52,0.63)$. In light of this result, specific MT effects on CD4 count were then examined separately, to account for the fact that the numerous immune system markers reported in those studies, and combined in the process of effect size calculation, might be obscuring this most important measure of disease progression in HIV+ persons. When an effect size for CD4 count only is generated from these two studies, the effect is only marginally larger and still non-significant $(g=0.24$, $95 \% \mathrm{CI}=-0.33,0.82$ ). Though these studies, and another study of MT for children with leukemia (29), make much of within-groups MT effects on immune system markers, the available between-groups data show that MT's effect on immune system function is probably small and possibly zero.

Pulmonary function. One study (No. 14 in Table 1) examined whether MT might generate an improvement in peak air flow for a sample of 20 children with cystic fibrosis. In comparison to a reading control group, the children receiving MT had higher peak air flow, $g=0.47$ (95\% CI $=-0.41$, $1.35)$, indicating MT may be of specific value to children with this condition. Another study (27) that does not permit effect size calculation reports multiple measures of breathing improvement for asthmatic children ages 6-8 years, though older children (ages 9-14 years) showed fewer improvements. Overall, evidence for MT effects on pulmonary function is promising, and further study is warranted.

Skin condition. Two RCT's examine MT's effect on skin condition. Children with atopic dermatitis were studied to determine the effect of MT on redness, scaling, lichenification, excoriation and pruritus (26). Though effect sizes cannot be calculated, study authors indicate some improvement following MT. Pediatric patients with hypertrophic scarring (HTS) were also studied and vascularity, pliability and height of the HTS revealed no appreciable effects. There is some evidence that pruritis decreased in certain patients with mature burn scars following MT. There is insufficient evidence to validate MT effects on skin condition in pediatric recipients.

Glucose level. (No. 7 in Table 1) One study of children with diabetes examined the effect of MT on blood glucose levels (25). The authors conclude that MT lowers mean blood glucose levels and that compliance for insulin and food regulation improved, though data necessary for effect size calculation are not reported.

\section{Behavioral Dimension}

Developmentally appropriate functioning. Two studies (No.'s 23 and 24 in Table 1) used the Developmental Programming for Infants and Young Children (DPIYC) scale to assess developmentally appropriate functioning in recipients following MT. The average effect was small and non-significant ( $g=0.24,95 \% \mathrm{CI}=-0.38,0.86$ ), though it must be pointed out that the studies had divergent results. Study number 24 yields a modest improvement in developmentally appropriate functioning in a sample of children with Down syndrome. Study number 23, with a sample of cerebral palsy sufferers, yields no effect. Possibly, these divergent results are the result of the different populations sampled within the studies. Taken together these results are inconclusive, but the encouraging results of the study with children who have Down syndrome indicate that further studies should be done with children having this or a related condition.

Spasticity. One study (No. 23 in Table 1), with a sample of children suffering from cerebral palsy, examined whether MT had an effect on spasticity by using the Spasticity scale/modified Ashworth scale. The small, non-significant effect $(g=0.26,95 \% \mathrm{CI}=-0.62,1.14)$ does not support an MT effect on spasticity. Because this study was very small ( $n=20)$, further testing in this area may be beneficial.

Hostility. One study (No. 21 in Table 1), with a small sample of aggressive adolescents, examined whether MT might reduce hostility compared with relaxation therapy. This study yields $g=-0.85(95 \% \mathrm{CI}=-1.85,0.15)$, a non-significant effect, but one that almost reaches significance in the wrong direction. The most tenable conclusion from this finding, a result based on a very small sample, is that MT has no effect on hostility beyond that provided by relaxation therapy to which it was compared. Though participants were randomly assigned, the MT group was approximately three points higher on the SCL-90R Hostility subscale prior to treatment. While scores decreased in the MT group during the treatment period, they also did in the relaxation therapy group, such that the MT group is still three points higher than the relaxation therapy group at the end of the treatment period. The authors' assertion that 'by the end of the study [massaged adolescents] reported feeling less hostile' obfuscates the fact that there was no between-groups effect. Related measures used in this study, such as the Overt Aggression Scale, were completed not by study participants, but by their legal guardians who were not blinded to group assignment, making their ratings prone to bias. Nevertheless, when all such measures included in the study are used to calculate an effect size, regardless of blinding, the result still converges on no effect.

Classroom behavior. One study (No. 13 in Table 1) examined the classroom behavior of students with attentiondeficit hyperactivity disorder using the Conners Teacher Rating Scale. Six factors of this scale were used including hyperactivity, conduct, emotional-indulgent, anxious-passive, asocial and daydream/attention problems. In comparison with a wait-list control, MT yielded $g=0.66$ (95\% CI $=-0.07$, 1.39), an effect that, while non-significant, is encouraging. Further studies of MT for this population are needed to confirm whether it might be of value in improving behavior, an outcome that seems possible given MT's relatively well-established potential to reduce anxiety. Studies that do not permit effect size calculation also suggest some 
improvements in classroom behavior following a course of MT. Improved classroom behavior and social relatedness are reported in studies of preschool children (40) and children with autism (41).

Cognitive performance. (No. 11 in Table 1) A study of MT for children in preschool concludes that MT impacts cognitive performance (42). This study used three subtests of the Wechsler Preschool and Primary Scale of IntelligenceRevised, but does not report sufficient data to quantify the effect.

Sleep and relaxation. (No.'s 1, 2 and 18 in Table 1) Though none permit effect size calculation, three studies have examined the effect of MT on sleep and relaxation. In one study, parents of children with autism recorded their children's sleep behavior in sleep diaries that included 5-point Likert scales (41). It is reported that the MT group showed decreases in fussing/restlessness, crying, self-stimulation behavior and getting out of bed. Nighttime sleep recordings were conducted for a previously mentioned study of MT for child and adolescent psychiatric patients (21). A video camera was set up on a tripod in the participant's room. The video tapes were subsequently coded for quiet sleep, active sleep, awake and lying quietly, and awake and active. Percentage of time asleep increased significantly from the first to last day of MT and the percentage of nighttime wakefulness decreased over the same time frame. Finally, children who experienced Hurricane Andrew were rated by an observer on a visual analogue scale (VAS), based on the child's apparent relaxation level (23). The VAS relaxation score increased significantly from the first to the last day of MT for the MT group. Possibly, MT promotes sleep and relaxation, but current studies do not permit this effect to be quantified.

\section{Discussion}

Available data reveals that MT provides benefit to pediatric recipients, though not as universally as has sometimes been reported. Benefits from both single-dose and multiple-dose sessions are evident. Most of the statistically significant effect sizes were observed for affective outcomes; findings for the behavioral and physiological dimensions were less consistent. These results parallel known MT effects in adult recipients, where multiple-dose reductions of depression and trait anxiety are the largest effects.

In reviewing MT for pediatric recipients, we encountered several weaknesses endemic to the MT research literature that should be addressed in subsequent studies. These included (i) low statistical power, (ii) frequent failure to report basic descriptive statistics, (iii) descriptions of results that do not logically follow study designs, and (iv) lack of replication. We discuss these in turn.

Low statistical power. Most pediatric MT studies were conducted with fewer than 30 participants. When studies are this small, only the largest effects have any likelihood of being uncovered. As researchers engaged in our own clinical MT studies, we are sensitive to the expense and difficulty involved in recruiting participants, but our empathy, unfortunately, does nothing to change the mathematics of the situation. The statistical power of MT studies must be increased (primarily by conducting studies with larger samples) if we wish to discover anything beyond the largest effects.

Failure to report basic descriptive statistics. Many pediatric MT studies have appeared in journals with lax standards for the reporting of data; there is no other explanation for why studies would fail to report the most basic descriptive statistics. When a report does not include the standard deviations that describe the spread of the data, that report has almost no value as scientific evidence. This must not be allowed to continue in MT research, because it represents an enormous waste of resources. Simply put, researchers who take the time, effort and expense to perform a study should not allow the results of that study to be published without the statistics that permit an understanding of the outcome. If a journal does not require them, then the onus is on the researcher to ensure that the statistics are reported.

Results that do not logically follow study designs. The importance of between-groups designs in MT has been noted (12). Why, then, do so many MT studies that employ a between-groups design emphasize within-group comparisons? The likely answer is that planned between-groups comparisons were non-significant (possibly due to low statistical power), so study authors may given in to the temptation to report the statistically significant, but misleading, within-group effect. This problem is rampant in MT research, and represents a real threat to the way this research will be perceived in the future. Many of the consumers of MT research are committed practitioners who have not had training in statistics and research design (though it must be noted that research literacy among MT practitioners is increasing). As such, they may be inclined to believe that coming across the sacrosanct ' $P<$ 0.05 ' is proof of an MT effect, without realizing that this 'effect' may be nothing more than the effect of time, a placebo effect and/or regression to the mean. Knowledgeable consumers of MT research must learn to distinguish within-group effects from between-groups effects, and MT researchers must clearly present between-groups findings when their studies employ a between-groups design.

Lack of replication. All but two of the pediatric MT studies we reviewed were conducted by the Touch Research Institute at the University of Miami. Their contribution to MT research has been considerable; however, scientific understanding is hampered when one laboratory is responsible for almost all of the results in an area of inquiry. Replication of results is a foundation of scientific progress, so it is necessary that other researchers contribute to this field. Given recent interest in CAM modalities, the small number of studies from other laboratories is surprising. We hope that this is about to change, and the success of the recent Highlighting Massage Therapy in CAM Research Conference (43) suggests that it will. Three pediatric MT RCT's from other research groups, currently in progress, support our optimism. Two of these examine MT for children and adolescents with cancer. While the results of 
a study that will examine pain, mood, stress and relaxation outcomes from MT and heat therapy for terminal children (44) cannot yet be reported, preliminary results of another study examining anxiety, fatigue, pain and nausea outcomes from MT in a sample of children with cancer (45) are promising; in particular, reductions of anxiety are expected to be consistent with what has been reported in this review. The third study, conducted by the first author of this review (S.B.) and researchers at the UCLA Pediatric Exercise Laboratory (46), evaluates pre-exercise MT for children with and without heart disease. Preliminary results show significant improvement in VO2 (oxygen consumption) for children who have an MT session prior to performing an exercise regimen on a stationary bicycle. If these results are validated in final analyses, they will be generally consistent with the finding that MT can improve pulmonary function. There are two other studies worth mentioning that have received IRB approval from Children's Memorial Hospital in Chicago and will be enrolling participants shortly. These studies are an evaluation of MT for reduction of pain, nausea and anxiety in pediatric cancer patients (47) and an evaluation of MT on immune function and heart rate variability in HIV-infected pediatric patients (48). The outcome measures for the first study are self-report ratings of pain, nausea and anxiety, whereas the second study evaluates T-Helper cells (CD4+), T-Suppressor cells (CD8+), Total B cells (CD3, CD19), Natural Killer cells (CD56+), viral load and heart rate variability.

\section{Conclusion}

Current research indicates that MT is not a panacea for conditions studied in the pediatric population. In contrast to what has sometimes been claimed, there is little to no evidence to date to support effects such as improved immune system functioning, reduction of spasticity, or amelioration of hostility. In addition, there is currently scant evidence that MT provides benefits by first reducing cortisol, as MT's effect on this stress hormone is seen to be small when analyzed correctly (i.e. in between-groups as opposed to within-group comparisons). There is, however, a set of MT effects that have been shown to have real value to the pediatric population. MT shows a considerable impact on the state and trait anxiety levels of children. Because these effects are strong, and also because they are consistent with the findings in adults, future research on the anxiolytic effects of MT on pediatric recipients does not need to simply replicate previous studies. The greatest progress can now be made by focusing on the mediators and moderators of MT effects on anxiety, and on testing explanatory theories of these outcomes. MT effects on arthritis pain and muscle tone also appear to be strong, but these results do need to be replicated, as they are based on single studies. Other pediatric outcomes that are promising, but in need of further study, include MT's effects on depression, negative mood, certain types of behavior (likely due to reductions of anxiety) and air flow in those suffering from pulmonary disorders such as cystic fibrosis. As increased statistical power in the form of additional studies is brought to bear on these potential benefits, it is likely that some will be quantitatively validated.

Finally, it has been noted that prior MT research has not accounted for the communication that inevitably takes place between massage therapists and their recipients, nor has it examined the likelihood that therapists and recipients develop a therapeutic relationship during the course of MT (10). This is also true in pediatric MT studies. MT has important parallels (in both process and outcomes) to psychotherapy (10), a treatment that relies on communication and therapeutic relationship to provide effects. It seems likely that MT effects, especially those belonging to the affective category, are mediated or moderated by these previously unexamined factors. These should not be neglected in subsequent pediatric MT research.

As adult consumers continue to explore and utilize all of their health care options, children will increasingly be recipients of MT. With this in mind, it is essential that we continue to study the benefits of MT for children, and the explanatory models that underlie them, so children's health and wellness can be maximized. The value of MT has been examined for many specific conditions that afflict children. It is our hope that this review has consolidated those findings, indicated areas that require further study, and led to an increased scientific understanding of pediatric MT.

\section{References}

1. Eisenberg DM, Davis RB, Ettern SL, Appel S, Wilkey S, Van Rompay M, et al. Trends in alternative medicine use in the United States, 1990-1997. JAMA 1998;280:569-75.

2. Loman DG. The use of complementary and alternative health care practices among children. J Pediatr Health Care 2003;17:58-63.

3. Sanders H, Davis MF, Duncan B, Meaney FJ, Haynes J, Barton LL. Use of complementary and alternative medical therapies among children with special health care needs in southern Arizona. Pediatrics 2003;111: 584-7.

4. Lin Y, Lee AC. Acupuncture for the Management of Pediatric Pain. Paper presented at Annual Meeting of the Pediatric Academic Societies, May 1999, San Francisco, CA.

5. Lin Y, Lee AC. Use of complementary and alternative medicine in pediatric pain services. Paper presented at Annual Meeting of the Pediatric Academic Societies, May 1999, San Francisco, CA.

6. Beider S. An ethical argument for integrated palliative care. Evid Based Complement Alternat Med 2005;2:227-31.

7. Ottenbacher KJ, Muller L, Brandt D, Heintzelman A, Hojem P, Sharpe P. The effectiveness of tactile stimulation as a form of early intervention: a quantitative evaluation. J Dev Behav Pediatr 1987;8:68-76.

8. Field T. Massage therapy. Med Clin North Am 2002;86:163-71.

9. Vickers A, Ohlsson A, Lacy JB, Horsley A. Massage for promoting growth and development of preterm and/or low birth-weight infants. The Cochrane Library, Issue 2. Chichester, UK: John Wiley \& Sons, Ltd, 2004.

10. Moyer CA, Rounds J, Hannum JW. A meta-analysis of massage therapy research. Psychol Bull 2004;130(1):3-18.

11. Tonelli MR, Callahan TC. Why alternative medicine cannot be evidencebased. Acad Med 2001;76:1213-20.

12. Field TM. Massage therapy effects. Am Psychol 1998;53:1270-81.

13. Rosenthal R. Writing meta-analytic reviews. Psychol Bull 1995;118: 183-92.

14. Retrieved from the AMTA website http://www.amtamassage.org/about/ definition.html. Accessed on March 1, 2006.

15. Borruat FX, Kawasaki A. Optic nerve massaging: an extremely rare cause of self-inflicted blindness. Am J Ophthalmol 2005;139:715-6. 
16. Teixeira CC, Dias RJ, Falcao-Reis FM, Santos M. Congenital dacryocystocele with intranasal extension. Eur J Ophthalmol 2005;15:126-8.

17. Juan $\mathrm{CH}$, Chu CC, Chang WK, Tsai SK. Recurrent life-threatening bradycardia in a child with retained tracheal stent. Paediatr Anaesth 2005; 15:251-3.

18. Field T, Lasko D, Mundy P, Henteleff T, Kabat S, Talpins S, et al. Brief report: autistic children's attentiveness and responsivity improve after touch therapy. J Autism Dev Disord 1997;27:333-8.

19. Hernandez-Reif M, Field T, Largie S, Hart S, Redzepi M, Nierenberg B, et al. Childrens' distress during burn treatment is reduced by massage therapy, (including commentary by Doctor ME). J Burn Care Rehabil 2001;22:191-5, 190 (16 ref.).

20. Lipsey MW, Wilson DB. Practical meta-analysis. Thousand Oaks CA: Sage, 2001.

21. Field T, Morrow CJ, Valdeon C, Larson S, Kuhn C, Schanberg S. Massage reduces anxiety in child and adolescent psychiatric patients. J Am Acad Child Adolesc Psychiatry 1992;31:125-31.

22. Spielberger CD. Conceptual and methodological issues in anxiety research. In: Spielberger CD (eds). Anxiety, vol. 2. Current Trends in Theory and Research. New York: Academic Press, 1972, 481-93.

23. Field T, Seligman S, Scafidi F, Schanberg S. Alleviating posttraumatic stress in children following Hurricane Andrew. J Appl Dev Psychol 1996; 17:37-50.

24. Field T, Grizzle N, Scafidi F, Schanberg S. Massage and relaxation therapies effects on depressed adolescent mothers. Adolescence 1996;31: $903-11$

25. Field T, Hernandez-Reif M, LaGreca A, Shaw K, Schanberg S, Kuhn C. Massage therapy lowers blood glucose levels in children with diabetes mellitus. Diabetes Spectrum 1997;10:237-9.

26. Schachner L, Field T, Hernandez-Reif M, Duarte AM, Krasnegor J. Atopic dermatitis symptoms decreased in children following massage therapy. Pediatr Dermatol 1998;15:390-5.

27. Field T, Henteleff T, Hernandez-Reif M, Martinez E, Mavunda K, Kuhn C, et al. Children with asthma have improved pulmonary functions after massage therapy. J Pediatr 1998;132:854-8.

28. Field T, Schanberg S, Huhn C, Field T, Fierro K, Henteleff T, et al. Bulimic adolescents benefit from massage therapy. Adolescence 1998;33: $555-63$.

29. Field T, Cullen C, Diego M, Hernandez-Reif M, Sprinz P, Kissell B, et al. Leukemia immune changes following massage therapy. J Bodywork Movement Ther 2001;5:271-4.

30. Watson D. Mood and temperament. New York: Guilford Press, 2000.

31. McNair DM, Lorr M, Droppleman LE. POMS-profile of mood states. Educational and Industrial Testing Service: San Diego, 1971.

32. Dickerson SS, Kemeny ME. Acute stressors and cortisol responses: a theoretical integration and synthesis of laboratory research. Psychol Bull 2004;130:355-91.

33. Edwards S, Clow A, Evans P, Hucklebridge F. Exploration of the awakening cortisol response in relation to diurnal cortisol secretory activity. Life Sci 2001;68:2093-103.

34. McGrath P, Johnson G, Goodman J, Schillinger J, Dunn J, Chapman J. CHEOPS: a behavioral scale for rating postoperative pain in children. Adv Pain Res Ther 1985;9:395-402.

35. Hernandez-Reif M, Field T, Largie S, Hart S, Redzepi M, Nierenberg B, et al. Childrens' distress during burn treatment is reduced by massage therapy. J Burn Care Rehabil 2001;22:191-5.

36. Platania-Solazzo A, Field T, Blank J, Seligman F, Kuhn C, Schanberg S, et al. Relaxation theapy reduces anxiety in child/adolescent psychiatry patients. Acta Paedopsychiatr 1992;55:115-20.
37. Ingram RE, Siegle GJ. Contemporary methodological issues in the study of depression: not your father's oldsmobile. In: Gotlib IH, Hammen CL (eds). Handbook of Depression. New York: Guilford Press, $86-114$.

38. Phillips BN, Martin RP, Meyers J. Interventions in relation to anxiety in school. In: Speilberger CD (eds). Anxiety, vol. 2. Current Trends in Theory and Research. New York: Academic Press, 1972, 409-64.

39. Field T, Quintino O, Hernandez-Reif M, Koslovsky G. Adolescents with attention deficit hyperactivity disorder benefit from massage therapy. Adolescence 1998;33:103-8.

40. Escalona A, Field T, Cullen C, Hartshorn K, Cruz C. Behavior problem preschool children benefit from massage therapy. Early Child Dev Care 2001;161:1-5.

41. Escalona A, Field T, Singer-Strunck R, Cullen C, Hartshorn K. Brief report: improvements in the behavior of children with autism following massage therapy. J Autism Dev Disord 2001;31:513-6.

42. Hart S, Field T, Hernandez-Reif M, Lundy B. Preschoolers' cognitive performance improves following massage. Early Child Dev Care 1998;143:59-64.

43. Retrieved from the Massage Therapy Foundation website http://www. massagetherapyfoundation.org/found_news_researchconference.html. Accessed on March 3, 2006

44. Retrieved from the NIH website. Study title Massage and Heat Therapies for Children at End of Life http://crisp.cit.nih.gov/crisp/crisp_lib.query. Accessed September 15th, 2006.

45. Post-White J, Fitzgerald M, Sencer S, Hooke C, Hannahan A. Massage therapy in childhood cancer (in review).

46. Beider S, Chang RK, Joshi M. Effect of pre-exercise massage on exercise performance and cardiopulmonary response in children with or without heart disease (In review).

47. Wang S. Evaluation of Massage Therapy for Reduction of Pain, Nausea, and Anxiety in Pediatric Cancer Patients (IRB Approved).

48. Wang S, Yogev R. Evaluation of Massage Therapy on Immune Function and Heart Rate Variability in HIV-infected Pediatric Patients (IRB Approved).

49. Hernandez-Reif M, Field T, Krasnegor J, Martinez E. Children with Cystic Fibrosis Benefit From Massage Therapy. J Pediatr Psychol 1999;24: $175-81$.

50. Jones NA, Field T. Massage and music therapies attenuate frontal EEG asymmetry in depressed adolescents. Adolescence 1999;34:529-34.

51. Patino O, Novick C, Merlo A, Benaim F. Massage in hypertrophic scars. $J$ Burn Care Rehabil 1998;19:268-71.

52. Diego MA, Field T, Hernandez-Reif M, Shaw K, Friedman L, Ironson G. HIV adolescents show improved immune function following massage therapy. Intern J Neuroscience 2001;106:35-45.

53. Diego MA, Field T, Hernandez-Reif M, Shaw JA, Rothe EM, Castellanos D, Mesner L. Aggressive adolescents benefit from massage therapy. Adolescence 2002;37:597-607.

54. Shor-Posner G, Miguez M, Hernandez-Reif M, Perez-Then E, Fletcher MA. Massage treatment in HIV-1 infected Dominican children: a preliminary report on the efficacy of massage therapy to preserve the immune system in children without antiretroviral medication. J Altern Complement Med 2004;10:1093-5.

55. Hernandez-Reif M, Field T, Largie S, Diego M, Manigat N, Seoanes J, Bornstein J. Cerebral palsy symptoms in children decreased following massage therapy. Early Child Dev Care 2005;175:445-456.

Received March 15, 2006; accepted August 26, 2006 


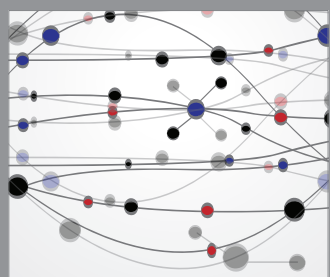

The Scientific World Journal
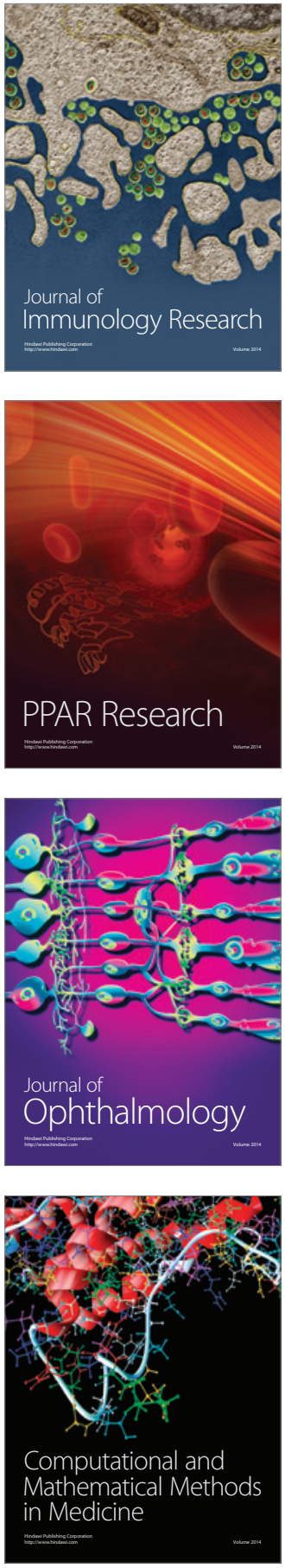

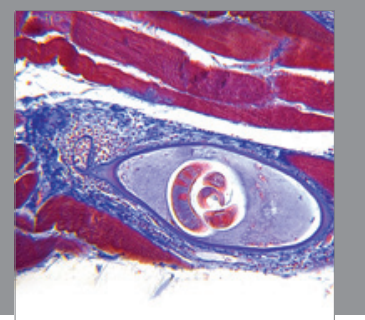

Gastroenterology

Research and Practice
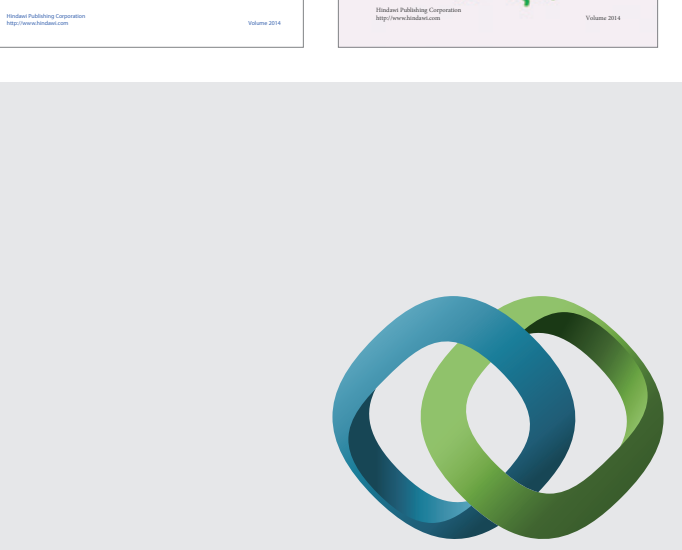

\section{Hindawi}

Submit your manuscripts at

http://www.hindawi.com
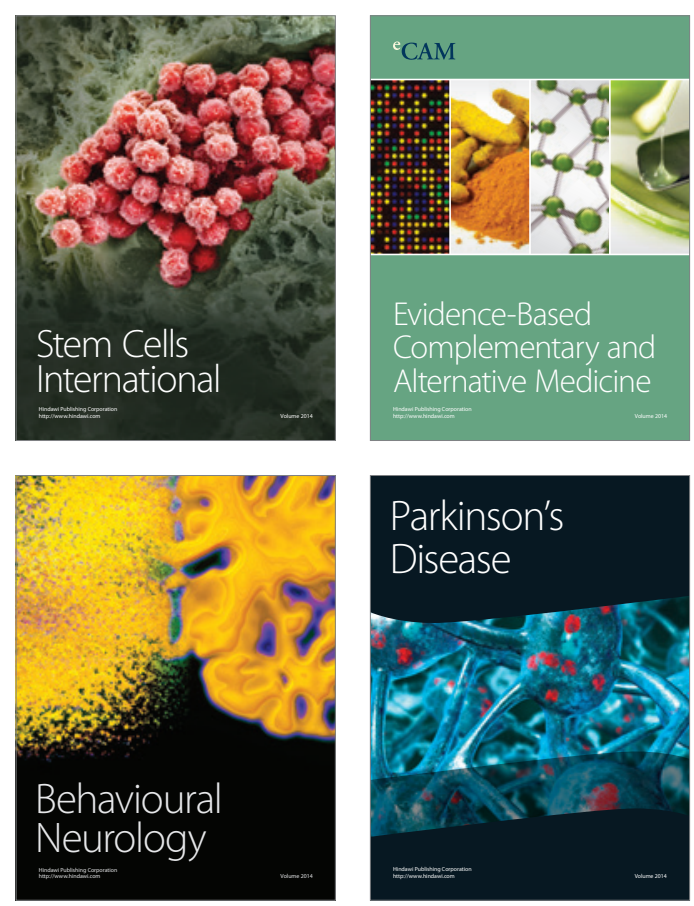

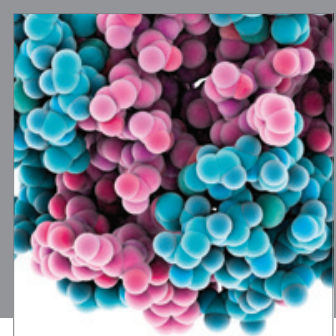

Journal of
Diabetes Research

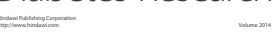

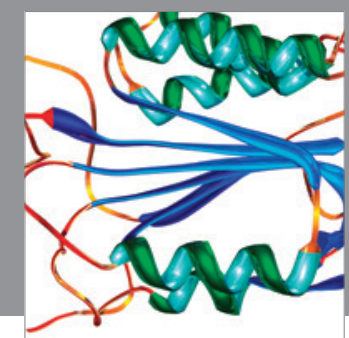

Disease Markers
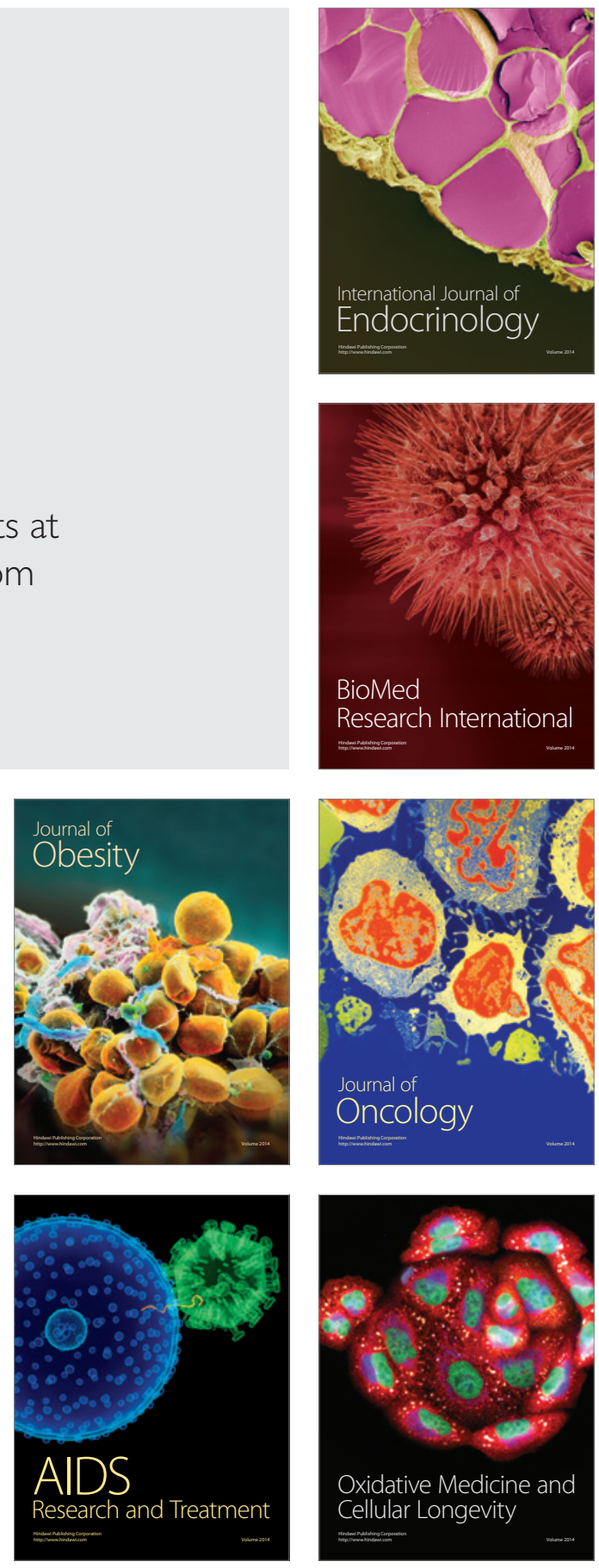